\title{
Immune-mediated necrotising myopathy is a rare statin-associated adverse effect: a case report
}

\author{
KF Lee *, FRCP, FHKAM (Medicine), Maria WH Mak, MRCP, FHKAM (Medicine), Virginia WN Lao, MRCP, FHKAM (Medicine), \\ Helen LK Yip, MRCP, FHKAM (Medicine), WY Lau, MRCP, FHKAM (Medicine), Victor TL Wong, MRCP, FHKAM (Medicine)
}

Department of Medicine and Geriatrics, Kwong Wah Hospital, Hong Kong

*Corresponding author: leekf1@ha.org.hk

Hong Kong Med J 2020;26:441-3

https://doi.org/10.12809/hkmj198226

\section{Case report}

The patient was a 60 -year-old woman with a 14-year history of type 2 diabetes mellitus and dyslipidaemia with a complication of background diabetic retinopathy. In December 2016, during a routine follow-up examination, the patient was found to have asymptomatic 5 -fold rise in liver aminotransferases. The patient's glycosylated haemoglobin level was 8.5\% and her low-density lipoprotein cholesterol $(\mathrm{LDL}-\mathrm{C})$ level was $2.0 \mathrm{nmol} / \mathrm{L}$. She was taking metformin $500 \mathrm{mg} 3$ times daily, gliclazide $160 \mathrm{mg}$ and vildagliptin $50 \mathrm{mg}$ twice daily, and atorvastatin $20 \mathrm{mg}$ once daily. In view of the possibility of statin-related hepatotoxicity, atorvastatin was withheld after 22 months of treatment. However, transaminitis persisted over the following 6 months after exclusion of viral hepatitis and any structural

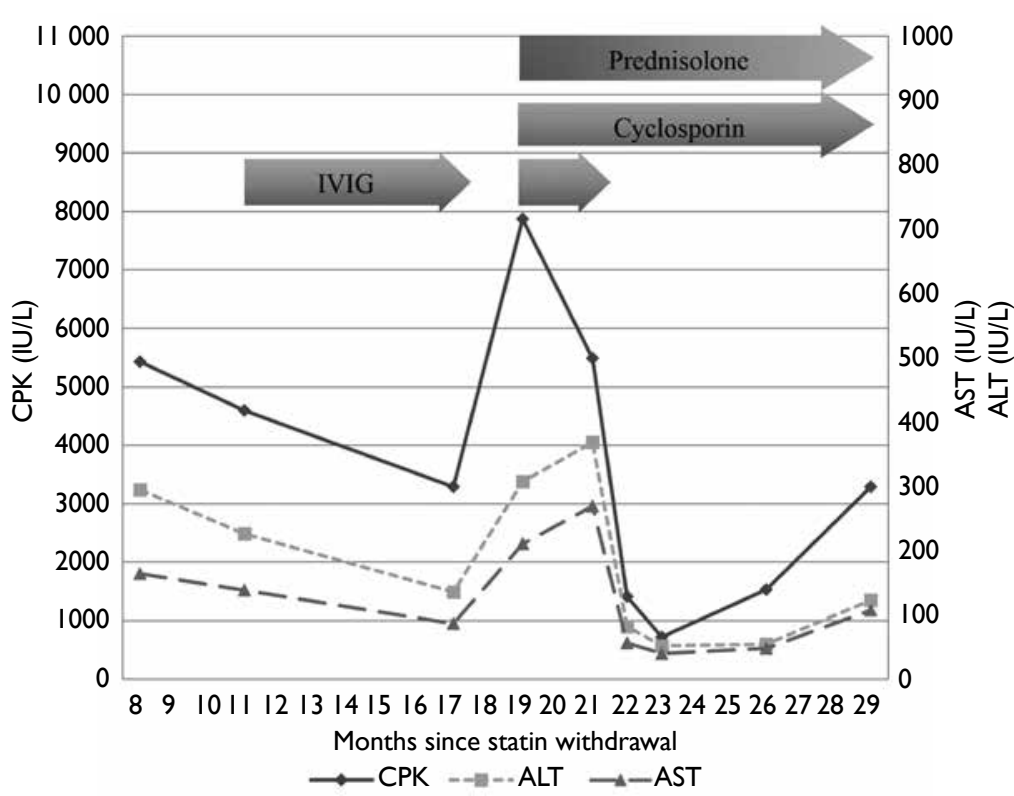

FIG. Clinical course and response to different modalities of treatment in a patient with immune mediated necrotising myopathy associated with statin use Abbreviations: $\mathrm{ALT}=$ alanine aminotransferase; $\mathrm{AST}$ = aspartate aminotransferase; $\mathrm{CPK}=$ creatine phosphokinase; $\mathrm{IVIG}=$ intravenous immune globulin abnormality. After 2 months, the patient complained of bilateral thigh weakness (Medical Research Council grade 4/5) and myalgia that prevented her from climbing stairs. Blood tests revealed elevated levels of creatinine kinase (CK) [5426 IU/L; normal range, 26-192 IU/L], alanine aminotransferase (294 IU/L; normal range, <47 IU/L), aspartate aminotransferase (164 IU/L; normal range, <36 IU/L), and lactate dehydrogenase (722 IU/L; normal range, 110-210 IU/L). Other inflammatory markers for myositis including anti-Jo-1 antibodies were normal. Urine for myoglobulin was negative and renal function was normal. With persistent clinical and biochemical abnormalities 9 months after statin cessation and no history of potential drug or health products that might induce myositis, immunemediated necrotising myopathy (IMNM) associated with statins was suspected. Electromyography suggested active myopathic changes while muscle biopsy revealed atrophy of multiple muscle fibres, necrosis and regeneration without inflammatory infiltrates. Diagnosis was finally confirmed following an enzyme-linked immunosorbent assay by a marked elevation of anti-3-hydroxy-3-methylglutaryl-CoA reductase (anti-HMGCR) autoantibody to $>200 \mathrm{IU} / \mathrm{mL}$ (normal range, $<20 \mathrm{IU} / \mathrm{mL}$ ). Considering her reasonable glycaemic control, monotherapy with intravenous immune globulin (IVIG) was initiated at a rate of $2 \mathrm{~g}$ per kilogram body weight per month. Muscle power increased and CK decreased (Fig) so IVIG was stopped after 6 months. Nonetheless her muscle weakness worsened and extended to involve the upper limbs as well as her ability to swallow, and CK rose from $3200 \mathrm{IU} / \mathrm{L}$ to almost $8000 \mathrm{IU} / \mathrm{L}$ after 2 months. High-dose glucocorticoids (intravenous methylprednisolone $500 \mathrm{mg} /$ day for 3 days followed by oral prednisolone $45 \mathrm{mg} /$ day) and cyclosporin were started. A monthly IVIG infusion was also added in the initial 2 months to enhance the therapeutic effect for her severe myopathy. After 4 months, her weakness improved and CK dropped below $1000 \mathrm{IU} / \mathrm{L}$. Owing to deteriorating glycaemic control (glycosylated haemoglobin level deteriorated to $9.1 \%$ ) and acute glaucoma, early tapering of glucocorticoid dose was considered. However, serum 
CK level returned to $2000 \mathrm{IU} / \mathrm{L}$ when prednisolone dose was weaned down to $7.5 \mathrm{mg}$ daily, although she retained full muscle power. Another steroidsparing agent, either methotrexate or rituximab, was considered.

\section{Discussion}

Statins are well-proven lipid-lowering drugs that reduce LDL-C and hence cardiovascular morbidity and mortality, in both primary and secondary prevention. Their use is recommended in a wide range of patients and high intensity therapy (LDL-C reduction $\geq 50 \%$ ) is indicated in a significant proportion. ${ }^{1}$

Despite their acceptable side-effect profile, about $10 \%$ of patients report statin-associated muscle symptoms (SAMS) such as myalgia and/or weakness. ${ }^{2}$ Toxic myopathy, defined as SAMS with marked elevation ( $>10$ times the upper limit of normal) of CK, occurs in approximately 1 in 10000 patients treated with statins per year. Typically, this condition remits spontaneously with cessation of statin use. On the contrary, statin-associated IMNM, a rarer adverse effect with an estimated occurrence of 2 to 3 per 100000 treated patients, is unlikely to be resolved by statin withdrawal, despite having similar SAMS and muscle enzyme increment. ${ }^{3}$ The IMNM was only suspected in our case 9 months after cessation of statin therapy, probably due to an initial lack of SAMS and misinterpretation that the elevated aminotransferases originated from the liver rather than muscle. It is important to also check CK in asymptomatic statin users with elevated aminotransferase levels to enable early diagnosis of statin-associated myopathy.

The IMNM is now recognised as a distinct form of myositis, usually presenting with symmetrical proximal arm or leg weakness with marked elevation of CK ( $>10$ times the upper limit of normal), muscle oedema, and atrophy on magnetic resonance imaging. In addition, muscle cell necrosis and regeneration along with minimal inflammatory infiltrates in muscle biopsy is evident and irritable myopathy on electromyography. ${ }^{3}$ Our patient had clinical features compatible with most of these symptoms. Unlike other SAMS phenotypes, there are no identifiable risk factors such as lipophilic (vs hydrophilic) statins, high-dose statins, old age, female gender, small body frame, liver or renal failure, and concomitant medications metabolised by the same hepatic P450 isoforms ${ }^{2}$ in statin-associated IMNM (Table). The detection of anti-HMGCR autoantibody in 2010 revolutionised the pathophysiology, diagnosis, disease classification, and treatment of this disease entity. This autoantibody detected by means of an enzyme-linked immunosorbent assay is both sensitive and specific; it has been detected in 24 of 26 patients $(92 \%)$ with a clinical presentation compatible with statin-associated IMNM although it has not been detected in statin-treated patients who do not have SAMS or self-limiting toxic myopathy. The overall specificity of the commercial enzymelinked immunosorbent assays for anti-HMGCR

TABLE. Clinicopathological characteristics of statin-associated toxic myopathy and immune-mediated necrotising myopathy

\begin{tabular}{|c|c|c|}
\hline & Toxic statin myopathy & IMNM associated with statin \\
\hline Estimated incidence (per year) & 1 in 10000 & $2-3$ in 100000 \\
\hline Onset & Within months after statin use & From 2 months to 10 years \\
\hline Predisposing factors & $\begin{array}{l}\text { - Old age } \\
\text { - Female } \\
\text { - Chronic renal or liver diseases } \\
\text { - High-dose statin } \\
\text { - Concomitant drugs metabolised by same } \\
\text { hepatic cytochrome P450 isoforms }\end{array}$ & HLA-DRB1*11:01 \\
\hline Presentation & Weakness, myalgia, or both & Weakness, myalgia, or both \\
\hline Creatinine kinase increment & $>10$ to 100 ULN & $>10$ to 100 ULN \\
\hline Muscle biopsy & Necrotic and regenerating muscle fibres & $\begin{array}{l}\text { Necrotic and regenerating muscle fibres } \pm \\
\text { sparse perivascular macrophages infiltration }\end{array}$ \\
\hline Anti-HMGCR autoantibodies & Negative & Positive in $>90 \%$ \\
\hline $\begin{array}{l}\text { Clinical and biochemical improvement after } \\
\text { statin discontinuation }\end{array}$ & Likely & Unlikely \\
\hline Treatment & Statin discontinuation & $\begin{array}{l}\text { Statin discontinuation and } \\
\text { immunosuppressants }\end{array}$ \\
\hline Re-challenge with another statin & Possible & Avoid \\
\hline
\end{tabular}

Abbreviations: anti-HMGCR = anti-3-hydroxy-3-methylglutaryl-CoA reductase; IMNM = immune-mediated necrotising myopathy; ULN = upper limit of normal 
autoantibody may be as high as 99.3\%. ${ }^{4}$ Nevertheless anti-HMGCR autoantibodies can also be detected in patients with IMNM who have an underlying malignancy or who are statin-naïve, particularly with more widespread use of anti-HMGCR autoantibody in patients with myopathy. With the detection of another autoantibody against a signal recognition particle, in 2017 the European Neuromuscular Centre classified IMNM into three subtypes: antisignal recognition particle myopathy, anti-HMGCR myopathy and antibody-negative IMNM. ${ }^{3}$ Although these subtypes share similar clinical features to those mentioned above, they differ in environmental risk factors, genetic risk factors, cancer risks, extramuscular manifestations, and response to different treatment modalities and prognoses.

Although spontaneous improvement after statin cessation has been reported in case studies, most patients with this condition require one to two immunosuppressive agents, usually in the form of high-dose glucocorticoids plus one of the following; methotrexate, azathioprine, mycophenolate mofetil or cyclosporine, for initial disease control.,5 The IVIG has also been used successfully as first-line monotherapy and it may be considered in those with pre-existing diabetes, as in our patient. ${ }^{6}$ However, incomplete normalisation of $\mathrm{CK}$ and the need for a prolonged course of treatment suggests its inability to completely abolish the pathophysiological process that causes muscle damage. This was illustrated in our patient with a rebound in CK level 2 months after completion of a 6-month course of IVIG monotherapy. Rather, her condition stabilised following treatment with two immunosuppressants, prednisolone and cyclosporine, although her anti-glycaemic treatment needed to be intensified. Similar to the clinical course of other reported series, her condition relapsed upon weaning of glucocorticoid dosage. ${ }^{7}$ Apart from escalation of steroid dosage, other steroid sparing agents may need to be considered. Rituximab has emerged as a promising rescue agent in this situation. ${ }^{8}$ Lastly, as statin is a known trigger of anti-HMGCR autoantibody, re-challenge with any statin should be avoided and an alternative cholesterol-lowering agent such as ezetimibe or PCSK9 inhibitors can be considered. ${ }^{9}$

In conclusion, IMNM can occur rarely in patients who present with SAMS. Unlike toxic myopathy, clinical and biochemical abnormalities persist upon statin withdrawal and immunosuppressants are usually required for disease control.

\section{Author contributions}

All authors contributed to the concept or design of the study, acquisition of the data, analysis or interpretation of the data, drafting of the manuscript, and critical revision of the manuscript for important intellectual content. All authors had full access to the data, contributed to the study, approved the final version for publication, and take responsibility for its accuracy and integrity.

\section{Conflicts of interest}

All authors have disclosed no conflicts of interest.

\section{Funding/support}

This case report received no specific grant from any funding agency in the public, commercial or not-for-profit sectors

\section{Ethics approval}

The patient was treated in accordance with the Declaration of Helsinki. The patient provided written informed consent for all treatments and procedures.

\section{References}

1. Grundy SM, Stone NJ, Bailey AL, et al. 2018 AHA/ ACC/AACVPR/AAPA/ABC/ACPM/ADA/AGS/ APhA/ ASPC/NLA/PCNA Guideline on the Management of Blood Cholesterol: A report of the American College of Cardiology/American Heart Association Task Force on Clinical Practice Guidelines. Circulation 2019;139:e1082143.

2. Ward NC, Watts GF, Eckel RH. Statin toxicity. Circ Res 2019;124:328-50.

3. Pinal-Fernandez I, Casal-Dominguez M, Mammen AL. Immune-mediated necrotizing myopathy. Curr Rheumatol Rep 2018;20:21.

4. Mammen AL. Statin-associated autoimmune myopathy. N Engl J Med 2016;374:664-9.

5. Tiniakou E, Christopher-Stine L. Immune-mediated necrotizing myopathy associated with statins: history and recent developments. Curr Opin Rheumatol 2017;29:60411.

6. Mammen AL, Tiniakou E. Intravenous immune globulin for statin-triggered autoimmune myopathy. N Engl J Med 2015;373:1680-2.

7. Ramanathan S, Langguth D, Hardy TA, et al. Clinical course and treatment of anti-HMGCR antibodyassociated necrotizing autoimmune myopathy. Neurol Neuroimmunol Neuroinflamm 2015;2:e96.

8. Allenbach Y, Mammen AL, Benveniste O, Stenzel W; Immune-Mediated Necrotizing Myopathies Working Group. 224th ENMC International Workshop: Clinicosero-pathological classification of immune-mediated necrotizing myopathies Zandvoort, The Netherlands, 14-16 October 2016. Neuromuscul Disord 2018;28:87-99.

9. Albayda J, Christopher-Stine L. Identifying statinassociated autoimmune necrotizing myopathy. Cleve Clin J Med 2014;81:736-41. 